\title{
Thermodynamics and structural properties of the dipolar Yukawa fluid
}

\author{
István Szalai ${ }^{\text {a) }}$ \\ Department of Chemistry, The University of Hong Kong, Pokfulam Road, Hong Kong \\ Douglas Henderson ${ }^{\text {b) }}$ and Dezsö Boda ${ }^{\mathrm{c})}$ \\ Department of Chemistry and Biochemistry, Brigham Young University, Provo, Utah 84602-5700 \\ Kwong-Yu Chan ${ }^{\text {d) }}$ \\ Department of Chemistry, The University of Hong Kong, Pokfulam Road, Hong Kong
}

(Received 27 January 1999; accepted 5 April 1999)

\begin{abstract}
We report computer simulations and a theoretical study of the thermodynamics and structure of a dipolar Yukawa system. A comparison between the analytical mean spherical approximation (MSA) solution, perturbation theory and Monte Carlo simulation data of pressure, internal energy and dielectric constant is given. In the perturbation theory, the MSA solution of hard core Yukawa fluid is used as a reference system. It was found that the MSA solution is reasonable only at lower dipole moments, while the perturbation theory gives good results at low and high values of dipole moment. Liquid-vapor coexistence data of dipolar Yukawa fluid are also obtained by Monte Carlo simulation and by both MSA and perturbation theory. It was found that at high dipole moments the liquidvapor equilibrium disappears while chain-like structures appear in the low density fluid phase. The appearance of chain-like structures of dipolar Yukawa fluid is discussed in comparison with the Stockmayer fluid. (C) 1999 American Institute of Physics. [S0021-9606(99)50225-0]
\end{abstract}

\section{INTRODUCTION}

The understanding of the structures and phase transitions of simple polar systems has changed dramatically in recent years. The simplest available description of a polar liquid at the molecular level is the well known dipolar hard sphere (DHS) fluid model. In connection with this fluid, one of the most surprising recent observations is the lack of a liquidvapor phase transition. ${ }^{1-3}$ This lack of the liquid-vapor equilibrium is explained by the formation of chain-like structures in the low density DHS fluid phase. It seems that a minimal amount of dispersion interaction is necessary to stabilize the liquid-vapor equilibrium; although, in the case of dipolar hard spherocylinders, a liquid-vapor coexistence island was found by the Gibbs ensemble simulation method ${ }^{4}$ in a system without such a dispersion force. From the point of view of the liquid-vapor phase transition of a real substance, the Stockmayer (STM) model is more reasonable ${ }^{5,6}$ than the DHS, but it does not have an analytical solution in the framework of any known theory. Recently, both the DHS and STM fluids have been used intensively as a reference system to the calculation of isotropic-nematic and isotropicferroelectric phase transitions of dipolar nonspherical particles. For the calculation of the isotropic-nematic phase transition of dipolar hard spherocylinders, a generalization of the perturbation theory for dipolar hard spheres, proposed by

\footnotetext{
${ }^{a)}$ Permanent address: Department of Physical Chemistry, University of Veszprém, H-8201 Veszprém, PO Box 158, Hungary. Electronic mail: szalai@almos.vein.hu

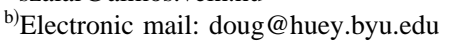

${ }^{c}$ Permanent address: Department of Physical Chemistry, University of Veszprém, H-8201 Veszprém, PO Box 158, Hungary. Electronic mail: boda@almos.vein.hu

${ }^{d)}$ Author to whom correspondence should be addressed. Electronic mail: hrsccky@hku.hk
}

Larsen $e t$ al.,${ }^{7}$ has been given by Williamson and del Rio. ${ }^{8}$ Density functional theoretical applications were proposed by Groh and Dietrich ${ }^{9,10}$ to study the phase transitions of spherical and nonspherical dipolar particles. Also, phase transitions in dipolar fluids have been investigated by integral equations using the reference hypernetted chain approximation. ${ }^{11}$ With the lack of a liquid-vapor phase transition, the DHS model, as a reference system, cannot predict the complex phase equilibrium curves. These facts justify that a new, more realistic analytically solvable dipolar fluid model may be of interest in the statistical mechanics of phase transitions of polar fluids.

In our previous paper, ${ }^{12}$ in the framework of the mean spherical approximation (MSA), an analytical solution has been given for the hard core dipolar Yukawa (DY) fluid. It has been shown that the thermodynamical properties of DY fluid can be calculated easily from the corresponding results of hard core Yukawa ${ }^{13}$ and DHS ${ }^{14}$ fluids. We note, that the DY potential proved to be successful in the perturbation theory study of phase separation of polar component fluid mixtures in interfaces. ${ }^{15}$ In the present paper, a comparison of thermodynamic and dielectric data for DY fluid is given between Monte Carlo (MC) and MSA calculation. Using the MSA pair correlation functions of the hard core Yukawa fluid, a perturbation theory description for the DY fluid is proposed. The liquid-vapor phase equilibrium of this system and the formation of chain-like structures is also studied in detail.

\section{THE MODEL POTENTIAL}

The DY potential in Cartesian form is defined as

$u\left(\mathbf{r}_{12}, \mathbf{n}_{1}, \mathbf{n}_{2}\right)=u_{Y}\left(r_{12}\right)+u_{\mathrm{DD}}\left(\mathbf{r}_{12}, \mathbf{n}_{1}, \mathbf{n}_{2}\right)$, 
where

$$
u_{Y}\left(r_{12}\right)=\left\{\begin{array}{l}
\infty, \quad r_{12}<\sigma \\
-\frac{\varepsilon_{Y} \sigma}{r_{12}} \exp \left[-\lambda\left(r_{12}-\sigma\right)\right], \quad r_{12} \geqslant \sigma
\end{array}\right.
$$

is the Yukawa potential and

$$
u_{\mathrm{DD}}\left(\mathbf{r}_{12}, \mathbf{n}_{1}, \mathbf{n}_{2}\right)=-\frac{\mu^{2}}{r_{12}^{3}} D\left(\mathbf{n}_{12}, \mathbf{n}_{1}, \mathbf{n}_{2}\right)
$$

is the dipole-dipole interaction potential, where

$$
D\left(\mathbf{n}_{12}, \mathbf{n}_{1}, \mathbf{n}_{2}\right)=3\left(\mathbf{n}_{1} \cdot \mathbf{n}_{12}\right)\left(\mathbf{n}_{2} \cdot \mathbf{n}_{12}\right)-\left(\mathbf{n}_{1} \cdot \mathbf{n}_{2}\right),
$$

gives the rotational invariant angular dependence of the dipole-dipole interaction. Here $\varepsilon_{Y}$ and $\lambda$ specify the depth and range of the dispersion interaction, $\sigma$ is the diameter of hard core, $\mathbf{n}_{i}=\boldsymbol{\mu}_{i} / \mu$ and $\mathbf{n}_{12}=\mathbf{r}_{12} / r_{12}$ are unit vectors along $\boldsymbol{\mu}_{i}$ and $\mathbf{r}_{12}$, and $\mu$ and $r_{12}$ are the magnitudes of dipole moment and interparticle distance, respectively. Considering the thermodynamical properties, the Yukawa fluid mimics the Lennard-Jones (LJ) fluid when $\lambda=1.8 / \sigma$ and, therefore, the DY fluid is similar to the STM fluid in this case. In this work, all results are obtained with this value of $\lambda$.

\section{THERMODYNAMIC DESCRIPTION OF DY FLUID}

\section{A. Mean spherical approximation}

In our previous paper ${ }^{12}$ it has been shown that in the MSA solution of the DY fluid model the radial distribution function and the angular correlation functions can be decoupled. Therefore, the MSA free energy of the dipolar Yukawa fluid $\left(A_{\mathrm{DY}}^{\mathrm{MSA}}\right)$ can be expressed as

$$
A_{\mathrm{DY}}^{\mathrm{MSA}}=A_{Y}^{\mathrm{MSA}}+A_{\mathrm{DD}}^{\mathrm{MSA}},
$$

where $A_{Y}^{\mathrm{MSA}}$ is the free energy of the hard core Yukawa fluid and $A_{\mathrm{DD}}^{\mathrm{MSA}}$ is the excess free energy of the dipolar hard sphere fluid (of course, both in the framework of MSA). In the following, we assume that $A_{Y}^{\mathrm{MSA}}$ contains the ideal gas and the Carnahan-Starling hard sphere contributions. The analytic solution of MSA for the hard core Yukawa fluid was obtained by Waisman. ${ }^{13}$ To avoid repetition, the prediction of its thermodynamic properties are not discussed here. The free energy expression for the dipole-dipole interaction is known from Wertheim's ${ }^{14}$ analytical solution as

$$
\frac{A_{\mathrm{DD}}^{\mathrm{MSA}}}{N k T}=-\frac{3}{\eta} I(y),
$$

where $\eta=\pi \rho \sigma^{3} / 6$ is the packing fraction and $y$ $=4 \pi \rho \mu^{2} /(9 k T)$ is the dipole-strength function. In both quantities, $\rho=N / V$ means the number density, where $N$ is the number of particles and $V$ is the volume of the system. In the definition of $y, T$ and $k$ denote the temperature and the Boltzmann constant, respectively. The function, $I(y)$ is defined as

$$
I(y)=\frac{8}{3} \xi^{2}\left[\frac{(1+\xi)^{2}}{(1-2 \xi)^{4}}+\frac{(2-\xi)^{2}}{8(1+\xi)^{4}}\right],
$$

where $\xi$ is the solution of the following equation:

$$
\left[\frac{(1+4 \xi)^{2}}{(1-2 \xi)^{4}}-\frac{(1-2 \xi)^{2}}{(1+\xi)^{4}}\right]=3 y .
$$

Of course, via the dipole-strength function, the dipolar part of the free energy depends on the temperature, density, and dipole moment. The corresponding equations for the pressure and internal energy can be predicted from the free energy. ${ }^{12,16}$ In the next paragraph we give a perturbation theory approximation for the free energy of the DY fluid. It is worthwhile to extract the MSA results from the perturbation terms; therefore, we must know the power expansion of the $A_{\mathrm{DD}}^{\mathrm{MSA}}$ free energy term in terms of $y$. Using the power series expansion of Eqs. (7) and (8) on the basis of Eq. (6) we obtain that

$$
\frac{A_{\mathrm{DD}}^{\mathrm{MSA}}}{N k T} \simeq-\frac{3}{\eta}\left(\frac{1}{16} y^{2}-\frac{5}{256} y^{3}+\ldots\right) .
$$

\section{B. Perturbation theoretical approximation}

One of the most useful thermodynamical perturbation theories of polar fluids is based on the energy expansion $^{7,16-19}$ of the free energy that is an extension of the theory of Barker and Henderson. ${ }^{20,21}$ In this perturbation theory according to Eqs. (1)-(3) the Yukawa term can be considered as the reference potential and the dipole-dipole interaction is the perturbation potential. This division gives for the free energy of DY fluid that

$$
A_{\mathrm{DY}}=A_{Y}^{\mathrm{MSA}}+A_{1}+A_{2}+A_{3}+\ldots,
$$

where $A_{1}$ is the first order perturbation term, and so on. These terms can be calculated with the help of the distribution functions of the reference system. According to the symmetry of the dipole-dipole interaction potential the first order term vanishes. After some manipulation of the second order term we obtain:

$$
\frac{A_{2}}{N k T}=-\frac{9}{16} \frac{y^{2}}{\eta} I_{6}
$$

and

$$
I_{6}=\int_{0}^{\infty} \frac{g_{Y}\left(r_{12}\right)}{r_{12}^{4}} d r_{12},
$$

where $g_{Y}\left(r_{12}\right)$ is the pair correlation function of the Yukawa reference system. In our case the third order term is

$$
\frac{A_{3}}{N k T}=\frac{9}{32} \frac{y^{3}}{\eta} I_{d d d}
$$

and

$$
\begin{aligned}
I_{d d d}= & \int_{0}^{\infty} \int_{0}^{\infty} \int_{\left|r_{12}-r_{13}\right|}^{r_{12}+r_{13}} \frac{\left(1+\cos \alpha_{1} \cos \alpha_{2} \cos \alpha_{3}\right)}{r_{12}^{2} r_{13}^{2} r_{23}^{2}} \\
& \times g_{Y}\left(r_{12}, r_{13}, r_{23}\right) d r_{12} d r_{13} d r_{23} .
\end{aligned}
$$

where $g_{Y}\left(r_{12}, r_{13}, r_{23}\right)$ is the three-particle distribution function of the reference system and $\alpha_{i}$ is the interior angle at particle $i$ in the triangle formed by particles 1,2 , and 3 . The 
series expansion of Eq. (10) converges poorly. Following Larsen et al. ${ }^{7}$ and Rushbrooke et al., ${ }^{16}$ we use the corresponding Padé approximation:

$$
A_{\mathrm{DY}}=A_{Y}^{\mathrm{MSA}}+\frac{A_{2}}{1-A_{3} / A_{2}} .
$$

In the calculation of thermodynamic properties, we refer to this perturbation theory equation as PT1. For the case of charged hard sphere and dipolar hard sphere systems Henderson et al. ${ }^{22}$ have showed that the convergence of the perturbation series expansion is better if we use the DY MSA expression as the starting term, and remove the corresponding MSA terms from the perturbation approximation. In this case in third order formally we can write that

$$
A_{\mathrm{DY}}=A_{\mathrm{DY}}^{\mathrm{MSA}}+\widetilde{A}_{2}+\widetilde{A}_{3} .
$$

The $\widetilde{A}_{2}$ and $\widetilde{A}_{3}$ terms can be calculated by the help of Eqs. (9), (11), and (13):

$$
\frac{\widetilde{A}_{2}}{N k T}=-\frac{9}{16} \frac{y^{2}}{\eta}\left(I_{6}-\frac{1}{3}\right)
$$

and

$$
\frac{\widetilde{A}_{3}}{N k T}=\frac{9}{32} \frac{y^{3}}{\eta}\left(I_{d d d}-\frac{15}{72}\right) .
$$

By the help of the Padé approximation ${ }^{7,16}$ a further improvement can be reached in the convergence of the series:

$$
A_{\mathrm{DY}}=A_{\mathrm{DY}}^{\mathrm{MSA}}+\frac{\widetilde{A}_{2}}{1-\widetilde{A}_{3} / \widetilde{A}_{2}} .
$$

Hereinafter, we refer to this perturbation theoretical equation as PT2.

\section{DIELECTRIC CONSTANT OF DY FLUID}

\section{A. Mean spherical approximation}

It was shown ${ }^{12}$ that the dielectric constant of DY fluid is given by the formula due to Wertheim: ${ }^{14}$

$$
\varepsilon_{\mathrm{DY}}^{\mathrm{MSA}}=\frac{(1+4 \xi)^{2}(1+\xi)^{4}}{(1-2 \xi)^{6}},
$$

where $\xi$ can be calculated from Eq. (8). Of course the dielectric constant again depends on the $\rho, T$, and $\mu$ via the dipolestrength function. Similar to the free energy, the power series expansion of $\varepsilon$ with respect to $y$ reads as

$$
\varepsilon_{\mathrm{DY}}^{\mathrm{MSA}} \simeq 1+3 y+3 y^{2}+\frac{3}{16} y^{3}+\ldots .
$$

\section{B. Perturbation theoretical approximation}

In statistical thermodynamics the static dielectric constant is given by the Kirkwood equation ${ }^{19}$

$$
\frac{(\varepsilon-1)(2 \varepsilon+1)}{9 \varepsilon}=y\left(1+G_{1}\right),
$$

where the $G_{1}$ angular correlation parameter can be given by the pair correlation function of the system as

$$
G_{1}=\rho \int\left\langle\left(\mathbf{n}_{1} \cdot \mathbf{n}_{2}\right) g\left(\mathbf{r}_{12}, \mathbf{n}_{1}, \mathbf{n}_{2}\right)\right\rangle_{\omega_{1}, \omega_{2}} d \mathbf{r}_{12} .
$$

The designation $\langle\ldots\rangle_{\omega_{1}, \omega_{2}}$ denotes unweighted averaging over the orientations of dipoles 1 and 2. Similar to the dipolar hard sphere ${ }^{23}$ and Stockmayer ${ }^{24}$ fluids pair correlation function gives only a second order perturbation term contribution to the correlation parameter

$$
G_{1}=1+y^{2} \frac{9}{16 \pi^{2}} I_{d d \Delta},
$$

where

$$
\begin{aligned}
I_{d d \Delta}= & \frac{16 \pi^{2}}{2} \int_{0}^{\infty} \int_{0}^{\infty} \int_{\left|r_{12}-r_{13}\right|}^{r_{12}+r_{13}} \frac{\left(3 \cos ^{2} \alpha_{3}-1\right)}{r_{13}^{2} r_{23}^{2}} \\
& \times r_{12} g_{Y}\left(r_{12}, r_{13}, r_{23}\right) d r_{12} d r_{13} d r_{23} .
\end{aligned}
$$

In the case of dipolar hard sphere ${ }^{23}$ and Stockmayer ${ }^{24}$ systems, for the perturbation theoretical calculation of $\varepsilon$, the following power expansion of the Kirkwood equation proved to be more successful than the original Eq. (22):

$$
\varepsilon_{\mathrm{DY}}=1+3 y+3 y^{2}+3 y^{3}\left(\frac{9 I_{d d \Delta}}{16 \pi^{2}}-1\right) .
$$

One reason is that perturbation theory is exact in the first three terms in the density expansion of $\varepsilon$, but is only exact for the first term in the density expansion of the angular correlation parameter. The dielectric constant calculation Eq. (26) is referred to as PT1. Removing the corresponding MSA terms from the perturbation expansion of $\varepsilon$ we obtain that:

$$
\varepsilon_{\mathrm{DY}}=\varepsilon_{\mathrm{DY}}^{\mathrm{MSA}}+3 y^{3}\left(\frac{9 I_{d d \Delta}}{16 \pi^{2}}-\frac{17}{16}\right) .
$$

Hereinafter, we refer to this perturbation theoretical equation as PT2.

\section{COMPUTATIONAL DETAILS}

\section{A. Numerical calculations}

One of the most important features of the DY fluid is that an analytic solution is available in MSA. The solution of the MSA is analytic but implicit. In our previous paper ${ }^{12}$ it has been shown that the free energy of DY fluid is the sum that of the DHS and hard core Yukawa fluids in MSA. The numerical calculation of pressure and internal energy were carried out on the basis of the original works. ${ }^{13,14,16}$ To the calculation of the perturbation theoretical integrals $\left(I_{6}, I_{d d d}, I_{d d \Delta}\right)$ the knowledge of the MSA pair correlation function of the Yukawa fluid is necessary. It was calculated numerically on the basis of the Ornstein-Zernike equation, using the MSA direct correlation function and its analytically given Fourier transform. ${ }^{13,25}$ The details of this calculation are given in a previous paper of Henderson et al. ${ }^{26}$ The integral $I_{6}$ was calculated by the Simpson method. In connection with integrals $I_{d d d}$ and $I_{d d \Delta}$, we note that higher-body distribution functions are unknown even for hard sphere systems. However, they can be approximated using the superposition approximation

$$
g_{Y}\left(r_{12}, r_{13}, r_{23}\right) \simeq g_{Y}\left(r_{12}\right) g_{Y}\left(r_{13}\right) g_{Y}\left(r_{23}\right) .
$$


The integral $I_{d d d}$ was calculated by using the Simpson integrand method three times repeatedly. The integrand of $I_{d d \Delta}$ is long range, therefore it is difficult to obtain its values when the integrations are performed in a straightforward manner and no cutoff is used. In this work the values of $I_{d d \Delta}$ were calculated on the basis of the Fourier-transform convolution theorem proposed by Goldman ${ }^{24}$ and Tani et al. ${ }^{23}$ Of course, via the pair distribution functions all integrals depend on the density and temperature of the system. At a given temperature the liquid-vapor coexistence can be obtained by finding the intersection of the chemical potential versus pressure curves. The reduced chemical potential is given as

$$
\frac{\mu}{k T}=\frac{A}{N k T}+z
$$

where $z=p V /(N k T)$ is the compressibility factor and $p$ is the pressure, respectively. We note that at higher temperatures when in the two-phase region the singularity of the hard core Yukawa MSA solution is disappearing, ${ }^{27}$ the Maxwell "equal area" construction is also appropriate to obtain the equilibrium pressure and densities.

\section{B. Details of Monte Carlo simulations}

We present the simulation results of both canonical NVT and NpT ensembles using the usual Boltzmann sampling and periodic boundary conditions in a cubic box. A spherical cutoff of half the cell length was applied, and long range corrections (LRC) were taken into account. ${ }^{28}$ For the dispersion part of the potential, the usual Yukawa-tail LRC was used while for the dipole-dipole interaction a spherical reaction field LRC and a conducting boundary condition were applied. In both ensembles 256 particles were used. The simulations were started from an fcc lattice with randomly oriented dipoles. After 40000 equilibration cycles, $200000-$ 400000 production cycles were used. Statistical errors were calculated from the standard deviations of the subaverages containing 10000 cycles. The liquid-vapor equilibrium simulations were carried out using our extended NpT plus test particle method. ${ }^{29,30}$ The essential idea of the method is to choose a gas and a liquid point on the $(T, p)$ plane which are close enough to the vapor pressure curve and also to each other, calculate the chemical potential surfaces $\mu=\mu(T, p)$ in both phases in some neighborhood of these points, and search for the intersection of these surfaces, where the two chemical potentials are equal. The projection of this intersection to the $(T, p)$ plane gives a section of the vapor pressure curve, since the condition for intersection is the same as for the liquid-vapor equilibrium. The construction of the chemical potential surfaces can be carried out by using a Taylor expansion. The coefficients of the series expansion can be calculated on the basis of the Widom's test particle method and from fluctuation formulas. In our simulations, 256 real and 256 test particles were used. The length of the simulations was two to three times longer than the bulk phase simulations.

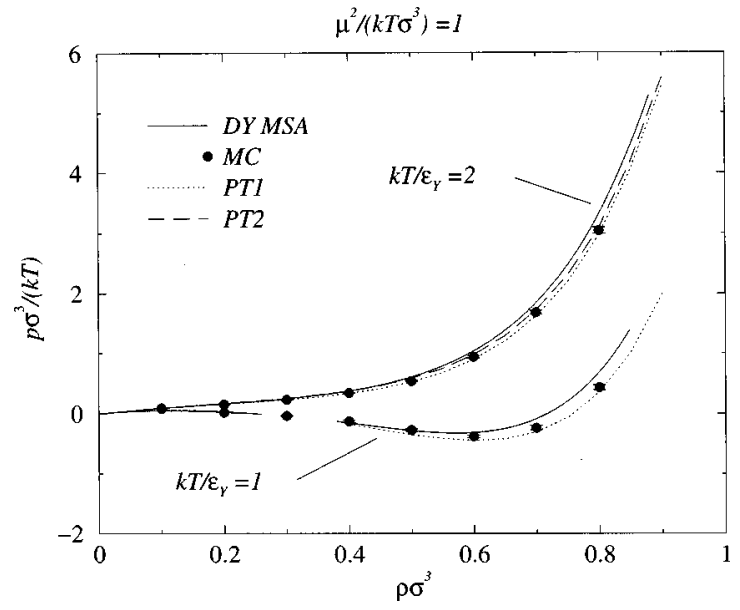

FIG. 1. The reduced pressure of the dipolar Yukawa fluid at a reduced dipole moment $\mu^{2} /\left(k T \sigma^{3}\right)=1$ calculated from the different theories as a function of the reduced density in comparison with simulation data. For $k T / \epsilon_{Y}=1$ the PT1 and PT2 curves coincide on the scale of the figure.

\section{RESULTS AND DISCUSSION}

The theoretical pressure isotherms (MSA and perturbation theory) at two different reduced dipole moments are compared to the corresponding MC simulation data in Figs. 1 and 2. At the lower temperature, due to the two-phase region, discontinuities can be found in both curves. At $\mu^{2} /\left(k T \sigma^{3}\right)=1$ the agreement between the MSA and simulation results is quite good at both temperatures. However, both perturbation approximations give slightly better results than the MSA. Considering the higher dipole moment (Fig. 2) the perturbation theory results are much more reliable than the corresponding MSA values. The fact that there is no large difference between the two perturbation theory results shows that the higher order terms in Eq. (9) do not make a substantial contribution to the free energy in MSA.

The density dependence of internal energy at different constant temperatures are displayed in Figs. 3 and 4. At the lower dipole moment the agreement between the MSA and

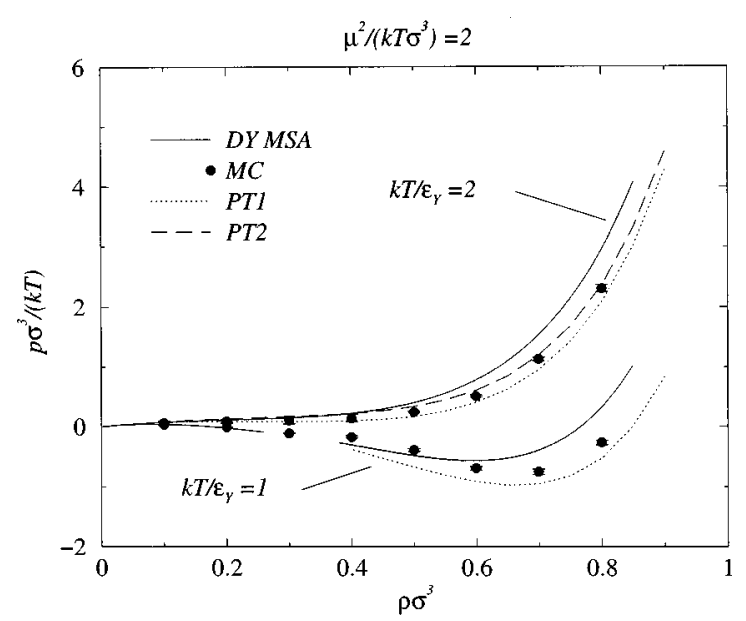

FIG. 2. The reduced pressure of the dipolar Yukawa fluid at a reduced dipole moment $\mu^{2} /\left(k T \sigma^{3}\right)=2$ calculated from the different theories as a function of the reduced density in comparison with simulation data. For $k T / \epsilon_{Y}=1$ the PT1 and PT2 curves coincide on the scale of the figure. 


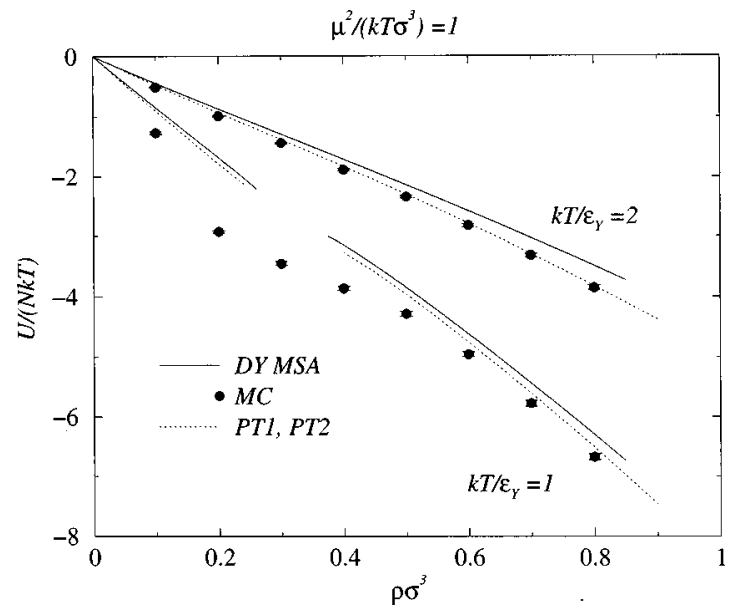

FIG. 3. The reduced internal energy of the dipolar Yukawa fluid at a reduced dipole moment $\mu^{2} /\left(k T \sigma^{3}\right)=1$ calculated from the different theories as a function of the reduced density in comparison with simulation data. The PT1 and PT2 curves coincide on the scale of the figure.

simulation results is reasonable, except in the vicinity of the two phase region. At both dipole moments, the perturbation theory results seem to be accurate except in the vicinity of the two phase region. The PT1 and PT2 approximations give practically the same results for the internal energy. It is not surprising that for the internal energy, the temperature derivative of the free energy, we get the same results in both approximations, because the $I_{6}$ and $I_{d d d}$ integrals depend weakly on temperature. (In the framework of the MSA the pair correlation function of Yukawa fluid is nearly independent of the temperature.)

The results of the theoretical calculations for the dielectric constant can be seen in Figs. 5 and 6 . At $\mu^{2} /\left(k T \sigma^{3}\right)$ $=1$, the agreement between MSA and simulation results is quite good, especially at lower densities. Figure 5 shows that PT2 gives the best prediction at that dipole moment. At the higher dipole moment, both MSA and PT2 underestimate the dielectric constant, but PT1 seems to be very good. In Figs. 5 and 6 the MC simulation data are obtained at two different

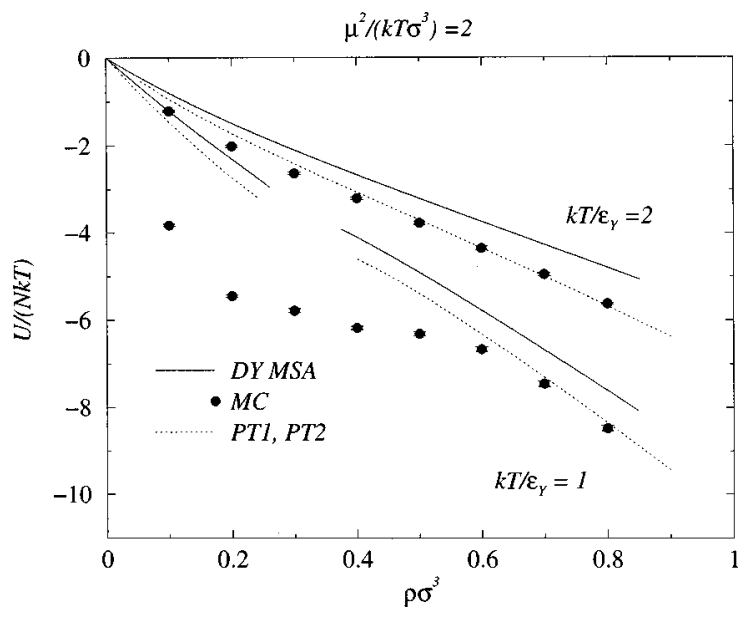

FIG. 4. The reduced internal energy of the dipolar Yukawa fluid at a reduced dipole moment $\mu^{2} /\left(k T \sigma^{3}\right)=2$ calculated from the different theories as a function of the reduced density in comparison with simulation data. The PT1 and PT2 curves coincide on the scale of the figure.

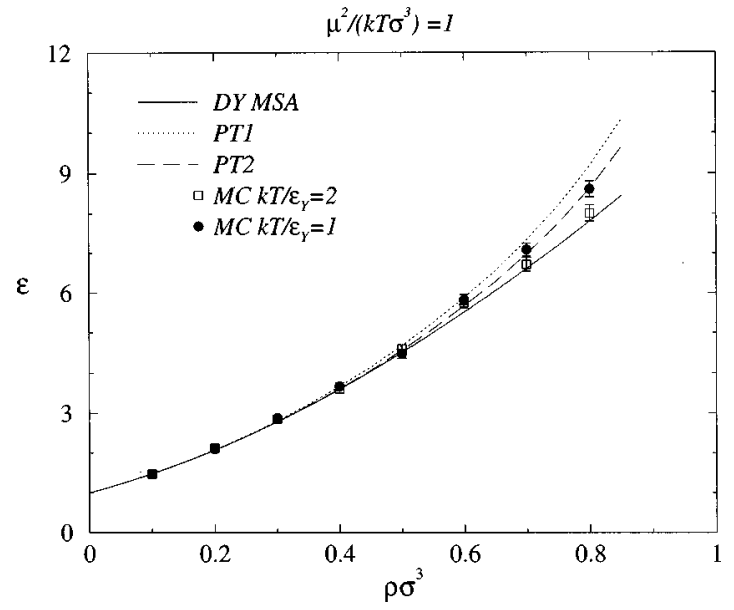

FIG. 5. The dielectric constant of the dipolar Yukawa fluid at a reduced dipole moment $\mu^{2} /\left(k T \sigma^{3}\right)=1$ calculated from the different theories as a function of the reduced density in comparison with simulation data.

temperatures. The MSA dielectric constant of DY fluid is the same as for the DHS and depends only on $y$; therefore, once $\mu^{2} /\left(k T \sigma^{3}\right)$ is fixed, $\varepsilon$ does not depend on temperature. Both perturbation theory approximations give a temperature dependence that are not visible in Figs. 5 and 6. We note that in the PT2, the dielectric constant of DY fluid is nearly the same as the corresponding value of the STM fluid, because the values of $I_{d d \Delta}$ integrals are very similar. (For example at $k T / \varepsilon_{Y}=k T / \varepsilon_{\mathrm{LJ}}=1$ and $\rho \sigma_{Y}^{3}=\rho \sigma_{\mathrm{LJ}}^{3}=0.8 I_{d d \Delta}=22.15$ for the DY fluid and $I_{d d \Delta}=22.27$ for the STM fluid. ${ }^{24}$ )

The MSA and perturbation theory liquid-vapor coexistence curves of DY fluid in comparison with simulation results are displayed in Fig. 7 at different reduced dipole moments. The agreement is reasonable only at low dipole moments. At higher dipole moments, the MSA underestimates the critical temperature while the perturbation theory overestimates the critical temperature. With increasing dipole moment we can see, that the estimated critical densities (from the simulation data) are lower. At a large dipole moment, $\left(\mu^{2} /\left(\varepsilon_{Y} \sigma^{3}\right)=16\right)$, we were not able to detect any

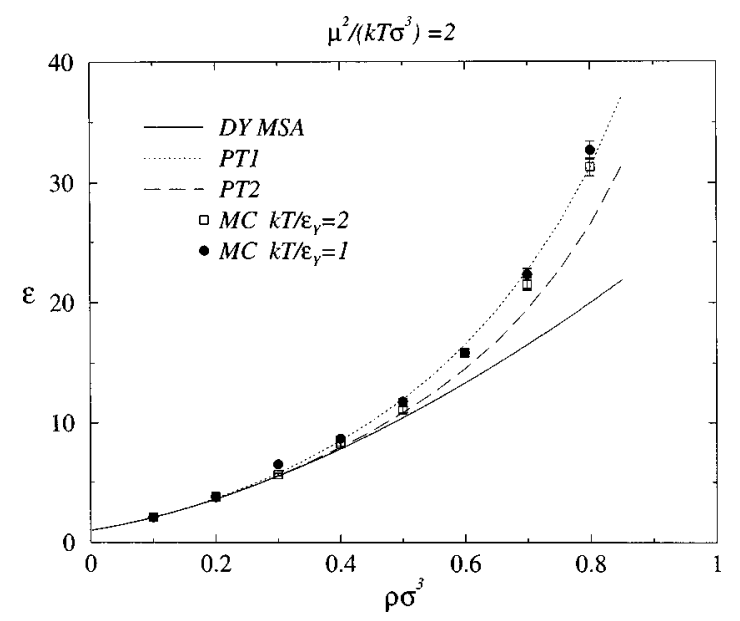

FIG. 6. The dielectric constant of the dipolar Yukawa fluid at a reduced dipole moment $\mu^{2} /\left(k T \sigma^{3}\right)=2$ calculated from the different theories as a function of the reduced density in comparison with simulation data. 


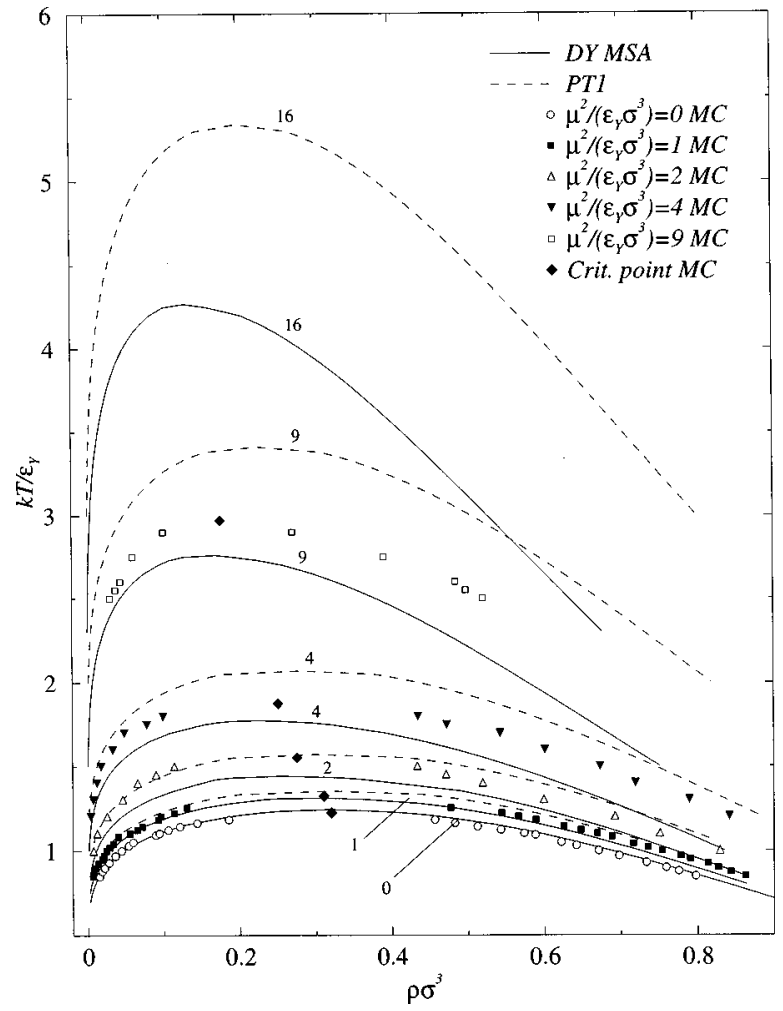

FIG. 7. Liquid-vapor coexistence curves for dipolar Yukawa fluids at different dipole moments calculated from MSA and perturbation theory in comparison with simulation data. The values of reduced dipole moment are denoted by the numbers at the theoretical curves.

liquid-vapor coexistence by the $\mathrm{NpT}$ plus test particle method. We can postulate that the liquid-vapor coexistence starts to disappear near this dipole moment.

The lack of the liquid-vapor equilibrium may be related to the formation of chains in the low-density gas phase. It was shown that in the presence of sufficient dispersion forces, for example, the LJ in STM fluid, no chain-like structures were found. Van Leeuwen and Smit ${ }^{2}$ have investigated the transition from a fluid with liquid-vapor coexistence to one without by modulating the range of the attractive part of the $\mathrm{LJ}$ force. They adjust a parameter $\lambda$ in the modified $\mathrm{LJ}$ equation,

$$
u\left(r_{12}\right)=4 \epsilon\left\{\left(\frac{\sigma}{r_{12}}\right)^{12}-\lambda\left(\frac{\sigma}{r_{12}}\right)^{6}\right\},
$$

which in effect is changing the strength of the LJ potential. It can be shown that Eq. (30) is equivalent to the ordinary LJ equation with $\sigma^{\prime}=\sigma \lambda^{-1 / 6}$ and $\epsilon^{\prime}=\epsilon \lambda^{2}$. Here, we would like to investigate the change of the fluid character from absence of chains to the presence of chains by slowly increasing the dipole moment relative to the dispersion force parameter $\epsilon_{Y}$. At low density, one can fix $T$ and $\epsilon_{Y}$ and increase $\mu$ to observe the appearance of chain-like structures. However, as the dipole moment increases, the critical temperature also increases, as shown in Figs. 7 and 8. Without a change in the value of $k T / \epsilon_{Y}$, an increase in the reduced dipole moment would result in a temperature much less then the critical temperature or perhaps even less than the triple point temperature. Thus, at increased reduced $\mu$ we must

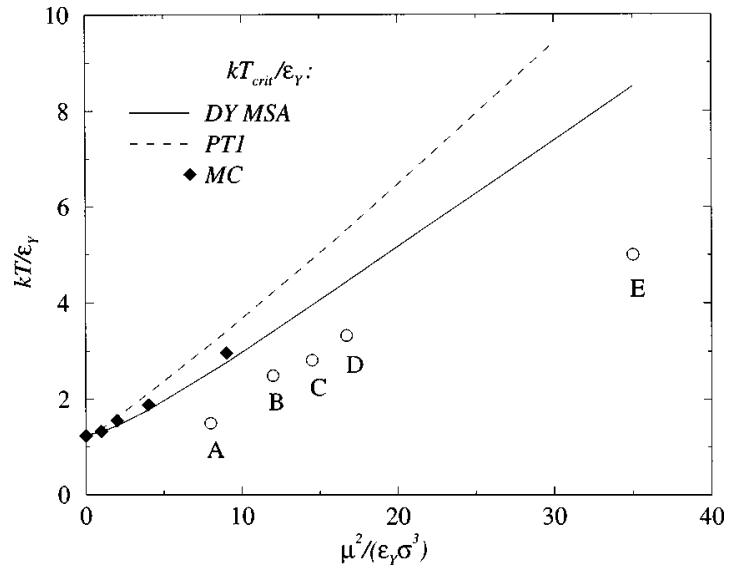

FIG. 8. The reduced critical temperature of dipolar Yukawa fluid as a function of the reduced dipole moment. For the meaning of the open circles, see the text.

examine greater values of the $k T / \epsilon_{Y}$; we adjust both $\epsilon_{Y}$ and $\mu$ so that $T / T_{\text {crit }}$, the reduced temperature, remains relatively constant.

A series of simulations were performed at $\rho \sigma^{3}=0.05$ following this scheme, with the path shown in Fig. 8. In points $\mathrm{A}-\mathrm{C}$ we were not able to detect any chain-like structure. The first chain-formation was found in the state point denoted by D. The corresponding snapshot of DY fluid is displayed in Fig. 9. The appearance of chains coincides with our NpT plus test particle simulation result of the absence of phase separation at $\mu^{2} /\left(\epsilon_{Y} \sigma^{3}\right)=16$. Upon further decrease of the relative strength of the dispersion interaction (in other words, increase both the dipole moment and temperature), the chain-like structure is more conspicuous. Figure 10 shows the appearance of longer chains in the simulation box. Continuing the path of Fig. 8, we will reach the DHS limit. We would like to confirm the appearance of chain-like structures in DHS. However, at $\mu^{2} /\left(\epsilon_{Y} \sigma^{3}\right)=\infty$, there is an arbitrary choice of dipole moment since $\epsilon_{Y}$ is zero. In the work of Van Leeuwen and Smit, they investigated the disappearance of the vapor-liquid coexistence by decreasing the dispersion force, rather than increasing the dipole moment as in

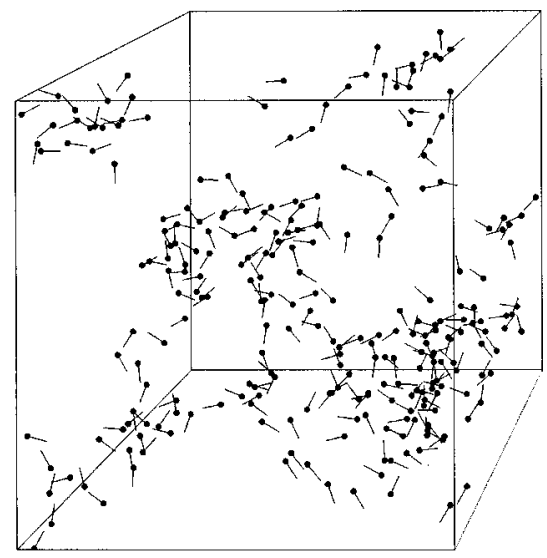

FIG. 9. Snapshot of the dipolar Yukawa fluid $\left(k T / \epsilon_{Y}=3.33, \rho \sigma^{3}=0.05\right.$ and $\left.\mu^{2} /\left(\epsilon_{Y} \sigma^{3}\right)=16.7\right)$. (Point D of Fig. 8.) 


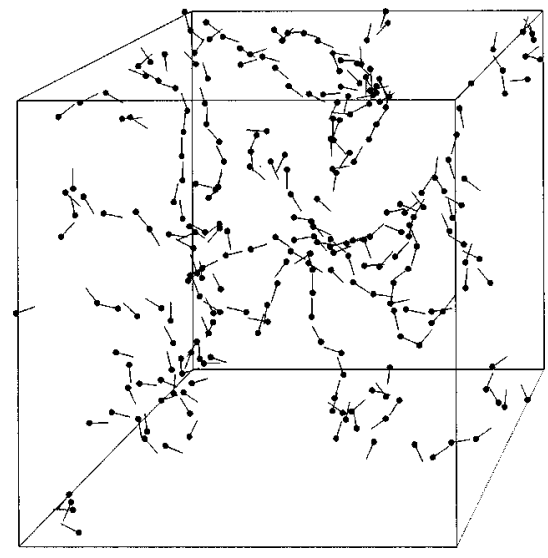

FIG. 10. Snapshot of the dipolar Yukawa fluid $\left(k T / \epsilon_{Y}=5, \rho \sigma^{3}=0.05\right.$ and $\mu^{2} /\left(\epsilon_{Y} \sigma^{3}\right)=35$ ). (Point E of Fig. 8.)

our study here. They did not find any liquid-vapor phase separation for $\lambda<0.3$, and concluded that the dipolar hard sphere and soft core fluids did not have liquid-vapor coexistence. In their study, they were not able to locate any phase separation for the values of $k T \sigma^{3} / \mu^{2} \leqslant 0.15$. As explained above, the potential of Eq. (30) is the Stockmayer potential. For $\lambda<0.3$, it corresponds to $\mu_{\mathrm{STM}}^{2} /\left(k T \sigma_{\mathrm{LJ}}^{3}\right) \geqslant 12$ in the ordinary STM fluid according to the above mentioned conversion of Eq. (30). To choose the value of dipole moment in DHS in the search for chains, we refer to this limit of $\mu^{2} /\left(k T \sigma^{3}\right)=12$, which corresponds to a highly polar DHS fluid. The simulation snapshot, which corresponds to this state is displayed in Fig. 11. The features of these chains are very similar to the partially flexible polymers and those obtained by Levesque and $\mathrm{Weis}^{31}$ for a little stronger dipolar hard spheres. The values of $\mu^{2} /\left(k T \sigma^{3}\right)$ in state points D and $\mathrm{E}$ are respectively, 5 and 7 . This means that the chain formation in a DY fluid started at higher temperatures than in the corresponding STM fluid. This difference is probably caused by the hard core of the Yukawa potential. Recently, Tavares et al. ${ }^{32}$ and Osipov et al..$^{33}$ reported density functional theory results for the critical locus of the STM fluid. They found that the critical density decreases with increasing dipolar strength. The relation of liquid-vapor coexistence

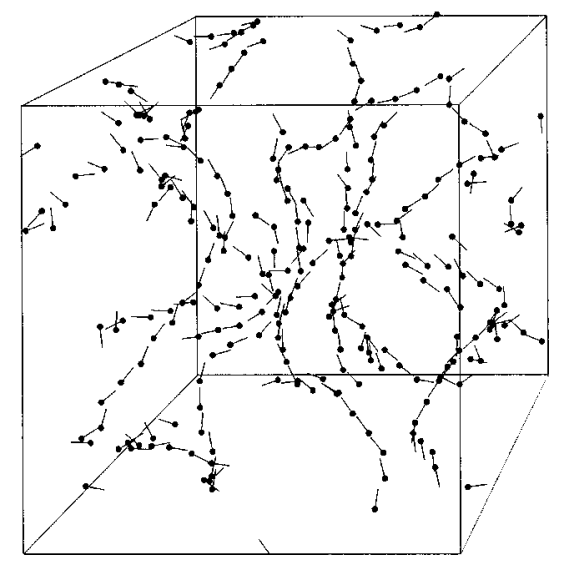

FIG. 11. Snapshot of the dipolar Yukawa fluid $\left(k T / \epsilon_{Y}=\infty, \rho \sigma^{3}=0.05\right.$, $\mu^{2} /\left(\epsilon_{Y} \sigma^{3}\right)=\infty$ and $\left.\mu^{2} /\left(k T \sigma^{3}\right)=12\right)$. (DHS fluid.) and chain-like structures were also discussed. It should be noted that our observations here are in good qualitative agreement with their results.

\section{SUMMARY AND CONCLUSIONS}

For the DY fluid, pressure, internal energy and dielectric constant as a function of density has been calculated at different temperatures and dipole moments by MSA, perturbation theory and MC simulation. At lower dipole moments, MSA results are in reasonable agreement with the corresponding MC simulation data. At higher dipole moments the perturbation theory gives unambigously better prediction. For the perturbation theory calculation, the hard core Yukawa MSA pair correlation function was used. Knowing the imperfection of this solution, better results might be obtained by using the exponential and linearized exponential modification of $\mathrm{MSA}^{26}$ pair correlation functions, but the available analytical expressions for the thermodynamic properties will be lost. The weakness of the MSA approximation mainly comes from the DHS MSA solution. Therefore, the combination of different routes of this part could improve the approximation as it was done in the case of STM fluid. ${ }^{34}$ It has been found that in the calculation of thermodynamic properties there is no significant difference between the two proposed perturbation theory approximations, although PT1 gives better prediction of the dielectric constant. Liquidvapor equilibrium simulations and both MSA and perturbation theory calculations have also been performed. The agreement between the simulation and theoretical data is reasonable only at low dipole moments. The disappearance of liquid-vapor coexistence and appearance of chain-like structure in the low density fluid phase are also studied. It has been found that chain formation started at lower reduced dipole moment, $\left(\mu^{2} /\left(k T \sigma^{3}\right)\right)$, than in the Stockmayer fluid. Even so, the value of this reduced dipole moment is still large. On the basis of its properties, the dipolar Yukawa model and its analytical MSA solution may be a valuable tool in the further research of structure and thermodynamics of dipolar fluids.

\section{ACKNOWLEDGMENTS}

The research was supported by the National Science Foundation (Grants CHE96-01971 and CHE98-13729), by the donors of the Petroleum Research Fund, administered by the American Chemical Society (Grant No. ACS-PRF 31573-AC9), by the Hungarian National Research Fund (OTKA-T025884), and by the Research Grants Council of Hong Kong (HKU249/95P).

${ }^{1}$ J. M. Caillol, J. Chem. Phys. 98, 9835 (1993).

${ }^{2}$ M. E. Van Leeuwen and B. Smit, Phys. Rev. Lett. 71, 3991 (1993).

${ }^{3}$ J. J. Weis and D. Levesque, Phys. Rev. Lett. 71, 2729 (1993).

${ }^{4}$ S. C. McGrother and G. Jackson, Phys. Rev. Lett. 76, 4183 (1996).

${ }^{5}$ M. E. Van Leeuwen, B. Smit, and E. M. Hendriks, Mol. Phys. 78, 271 (1993).

${ }^{6}$ M. E. Van Leeuwen, Fluid Phase Equilibria 99, 1 (1994).

${ }^{7}$ B. Larsen, J. C. Rasaiah, and G. Stell, Mol. Phys. 33, 987 (1977).

${ }^{8}$ C. D. Williamson and F. del Rio, J. Chem. Phys. 107, 9549 (1997).

${ }^{9}$ B. Groh and S. Dietrich, Phys. Rev. E 53, 2509 (1996).

${ }^{10}$ B. Groh and S. Dietrich, Phys. Rev. E 55, 2892 (1997). 
${ }^{11}$ S. Klapp and F. Forstmann, J. Chem. Phys. 106, 9742 (1997).

${ }^{12}$ D. Henderson, D. Boda, I. Szalai, and K. Y. Chan, J. Chem. Phys. 110, 7348 (1999).

${ }^{13}$ E. Waisman, Mol. Phys. 25, 45 (1973).

${ }^{14}$ M. S. Wertheim, J. Chem. Phys. 55, 4291 (1971).

${ }^{15}$ D. Henderson and W. Schmickler, J. Chem. Soc., Faraday Trans. 92, 3839 (1996).

${ }^{16}$ G. S. Rushbrooke, G. Stell, and J. S. Hoye, Mol. Phys. 26, 1199 (1973).

${ }^{17}$ G. Stell, J. C. Rasaiah, and H. Narang, Mol. Phys. 23, 393 (1972).

${ }^{18}$ G. Stell, J. C. Rasaiah, and H. Narang, Mol. Phys. 27, 1393 (1974).

${ }^{19}$ C. G. Gray and K. E. Gubbins, Theory of Molecular Fluids, Fundamentals (Clarendon, Oxford, 1984).

${ }^{20}$ J. Barker and D. Henderson, J. Chem. Phys. 47, 2856 (1967).

${ }^{21}$ J. Barker and D. Henderson, J. Chem. Phys. 47, 4714 (1967).

${ }^{22}$ D. Henderson, J. Barojas, and L. Blum, Rev. Mex. Fis. 30, 139 (1984).

${ }^{23}$ A. Tani, D. Henderson, J. A. Barker, and C. E. Hecht, Mol. Phys. 48, 863 (1983).
${ }^{24}$ S. Goldman, Mol. Phys. 71, 491 (1990).

${ }^{25}$ C. Hausleitner and J. Hafner, J. Phys. F: Met. Phys. 18, 1013 (1988).

${ }^{26}$ D. Henderson, E. Waisman, J. L. Lebowitz, and L. Blum, Mol. Phys. 35, 241 (1978).

${ }^{27}$ P. T. Cummings and E. R. Smith, Chem. Phys. 42, 241 (1979).

${ }^{28}$ M. P. Allen and D. J. Tildesley, The Computer Simulation of Liquids (Clarendon, Oxford, 1987).

${ }^{29}$ D. Boda, J. Liszi, and I. Szalai, Chem. Phys. Lett. 235, 140 (1995).

${ }^{30}$ D. Boda, J. Liszi, and I. Szalai, Mol. Phys. 85, 429 (1995).

${ }^{31}$ D. Levesque and J. J. Weis, Phys. Rev. E 49, 5131 (1994).

${ }^{32}$ J. M. Tavares, M. M. Telo da Gama, and M. A. Osipov, Phys. Rev. E 56, 6252 (1997); 57, 7367 (1998).

${ }^{33}$ M. A. Osipov, P. I. C. Teixeira, and M. M. Telo da Gama, Phys. Rev. E 54, 2597 (1997).

${ }^{34}$ G. Kronome, J. Liszi, and I. Szalai, J. Chem. Soc., Faraday Trans. 93, 3053 (1997). 